Representing uncertainty in the Rescorla-Wagner model: Blocking, the redundancy effect, and outcome base rate

Spicer, Stuart

http://hdl.handle.net/10026.1/17374

10.46221/ojepn.2021.6623

Open Journal of Experimental Psychology and Neuroscience Nurture Science Publishing Group

All content in PEARL is protected by copyright law. Author manuscripts are made available in accordance with publisher policies. Please cite only the published version using the details provided on the item record or document. In the absence of an open licence (e.g. Creative Commons), permissions for further reuse of content should be sought from the publisher or author. 


\title{
Representing uncertainty in the Rescorla-Wagner model: Blocking, the redundancy effect, and outcome base rate
}

\author{
Stuart G. Spicer* Andy J. Wills* Peter M. Jones* Chris J. Mitchell* Lenard Dome*
}

\begin{abstract}
It is generally assumed that the Rescorla and Wagner (1972) model adequately accommodates the full results of simple cue competition experiments in humans (e.g. Dickinson et al., 1984), while the Bush and Mosteller (1951) model cannot. We present simulations that demonstrate this assumption is wrong in at least some circumstances. The Rescorla-Wagner model, as usually applied, fits the full results of a simple forward cue-competition experiment no better than the Bush-Mosteller model. Additionally, we present a novel finding, where letting the associative strength of all cues start at an intermediate value (rather than zero), allows this modified model to provide a better account of the experimental data than the (equivalently modified) Bush-Mosteller model. This modification also allows the Rescorla-Wagner model to account for a redundancy effect experiment (Uengoer et al., 2013); something that the unmodified model is not able to do. Furthermore, the modified Rescorla-Wagner model can accommodate the effect of varying the proportion of trials on which the outcome occurs (i.e. the base rate) on the redundancy effect (Jones et al., 2019). Interestingly, the initial associative strength of cues varies in line with the outcome base rate. We propose that this modification provides a simple way of mathematically representing uncertainty about the causal status of novel cues within the confines of the Rescorla-Wagner model. The theoretical implications of this modification are discussed. We also briefly introduce free and open resources to support formal modelling in associative learning.
\end{abstract}

Keywords: associative learning, prediction error, uncertainty, modelling, blocking, redundancy effect, open science.

\section{Introduction}

Blocking (Kamin, 1969) is a type of cue competition that occurs when learning about one cue is apparently restricted by the simultaneous presence of another cue that has also been trained separately. For example, if a single cue is followed by an outcome $(\mathrm{A}+)$, and a separately-encountered compound containing that cue is followed by the same outcome $(\mathrm{AX}+)$, then learning about $\mathrm{X}$ is restricted (N.B. letters represent cues and + or - represents the presence or absence of the outcome). In humans, learning is often tested by asking participants to rate the likelihood of an outcome such as stomach ache, on the basis of specific cues such as foods (e.g. Jones et al., 2019). Participants rate blocked cues as a less likely cause of the outcome than an appropriate control (e.g. Y following B- BY+ training).

Early models of associative learning were unable to account for blocking. In particular, the associative learning model developed by Bush and Mosteller (1951) uses an individual prediction error, which means that any change in the strength of an association between a cue and an outcome is governed by the size of the error between the outcome that occurs and the outcome predicted by that cue alone. For

Copyright: (C) 2021. The authors license this article under the terms of the Creative Commons Attribution Share-Alike 4.0 License.

*School of Psychology, University of Plymouth, U.K. Email: stuart.spicer@plymouth.ac.uk example, if you predict that a certain type of food is safe to eat, but you subsequently suffer an allergic reaction after eating that food, then there will be a large prediction error. Once you have learned that this type of food is not safe to eat, there will be no error, and learning will be at asymptote. According to the Bush-Mosteller model, learning updates as follows:

$$
\Delta V_{x}=\alpha_{x} L\left(\lambda-V_{x}\right)
$$

In Equation 1, associative strength is denoted by $\mathrm{V}$, where $\Delta V_{x}$ is the change in associative strength for cue $\mathrm{X}$, and $V_{x}$ is the current associative strength of cue $\mathrm{X}$. The cue salience is represented by $\alpha$ and the outcome learning rate is represented by $L$. The asymptote of learning is represented by $\lambda$. The individual prediction error means that the model cannot account for blocking, as this effect is driven by the interference of simultaneously encountered cues. To overcome this, Rescorla and Wagner (1972) proposed a model with an overall prediction error, in which any change in associative strength is governed by the error between the outcome that occurs and the outcome predicted by all simultaneously present cues:

$$
\Delta V_{x}=\alpha_{x} L\left(\lambda-\Sigma V_{x}\right)
$$

The only change is that $\Sigma V$ has been incorporated as the overall associative strength of all simultaneously-present 
cues. The model can account for blocking because A takes up all the available associative strength on the A+ trials, meaning that there is no available associative strength for $\mathrm{X}$ to acquire on the $\mathrm{AX}+$ trials (assuming learning is at asymptote).

Dickinson et al. (1984) provided one of the first experimental demonstrations of blocking in humans. The Rescorla and Wagner (1972) model is widely assumed to adequately explain the full data of such experiments, whilst also providing a better account than Bush and Mosteller's (1951) model. In our first simulation, we conducted a model-fitting procedure on the full data of a simple forward cue competition blocking experiment (a standard design incorporating blocking, a control and filler cues), using both models. It is worth emphasising that we were exploring the ability of the models to account for the full set of experimental test cues, rather than just the blocked and control cues. A sufficiently adequate model should be able to account for a full experiment, rather than just accounting for an effect, which is only an extract of an experiment. Neither model provided an adequate account, with the overall fit of the Rescorla-Wagner model (to the data) being no better than Bush-Mosteller model.

\section{Simulation set 1: Blocking}

There are several simple human forward cue-competition experiments reported in the literature (e.g. Dickinson et al., 1984; Miller, 1996; Mitchell \& Lovibond, 2002), but none of these datasets have been made openly accessible. In order to provide an openly accessible dataset, we ran a standard forward cue competition experiment. The design included blocking $(\mathrm{A}+\mathrm{AX}+)$, a common control $(\mathrm{B}-\mathrm{BY}+)$, and filler cues intended to balance the number of cue types causing either stomach ache or no stomach ache (C- CD-). For brevity, details of all experiments and simulations are reported in Supplementary Materials (https://osf.io/7u6re/); this main article summarises the key findings.

A model fitting process was conducted on the data for all test stage cues, using standard implementations of the Bush and Mosteller (1951), and Rescorla and Wagner (1972) models. Following standard practice, we used gradient-descent optimisation to find the best-fitting parameters. This process involves exploring the parameter space, to minimise the difference (represented by the sum of squared errors) between the observed and predicted mean test ratings. It was necessary for each model to generate ratings consistent with the outcome-likelihood scale (between 0 and 10) used at test, rather than just output associative strengths (typically between 0 and 1). It is generally accepted that there is not a 1:1 linear relationship between associative strengths and responding (e.g. Gluck \& Bower, 1988), although as one increases so should the other. A standard solution to this problem is to use a logistic function to map associative strengths onto responses. The equation below is a logistic function suggested by Gluck and Bower:

$$
P_{x}=10 \frac{1}{1+e^{-\theta\left(V_{x}-\beta\right)}}
$$

$P_{x}$ denotes the simulated likelihood rating for cue $\mathrm{X}$, while $V_{x}$ denotes the associative strength. $\beta$ denotes the bias parameter for the output associative strength that will result in a rating of 5 (i.e. the middle of the scale). $\theta$ is a scaling parameter, where higher values mean that the function relating activation to rating becomes less linear and more logistic. As outlined, both models also require cue salience $(\alpha)$ and learning rate $(L)$ as their two standard parameters. Both of these parameters can have a value between 0 and 1 . Since these values are multiplied in the learning algorithm, the resulting product is necessarily a value between 0 and 1. For simplicity, these parameters were collapsed into a single learning rate parameter $(G)$. Therefore, the parameter space being explored consisted of $G, \beta$ and $\theta$. The value of each parameter was the same for all cues, since the counterbalancing of stimuli in the experimental design meant there was no theoretical basis for expecting these values to differ between cues. For these simulations, we defined a 'model' as a specific combination of processes and representational assumptions (see Wills \& Pothos, 2012). This includes the representation of stimuli within the model; in this case one cue per experimental stimulus. Additional cues can be included in some representations (e.g. to represent context). That was not our focus, but these concepts are considered further in the General Discussion.

\subsection{Results from standard model implemen- tations}

The best fitting model was the one that produced the smallest error between the predicted and observed test ratings. This was assessed using the mean error for each of the six test cues. Please note that (unlike the figures) these values are on a scale of 0-1 throughout this paper, consistent with associative strength. The adequacy of fit for each model was additionally assessed with the $R^{2}$ of the predicted versus observed ratings (where higher values indicate a better adequacy of fit). Both the Bush and Mosteller (1951) model, and the Rescorla and Wagner (1972) model produced a mean error of 0.08 and an $R^{2}$ of 0.71 . To put this latter figure in context, the best formal models of category learning produce $R^{2}$ values exceeding .95 for standard results in the field, with models that are clearly and ordinally wrong still sometimes producing $R^{2}$ values exceeding 0.85 (Nosofsky et al., 1994). On this basis, both models provide quite poor accounts of this basic blocking experiment. 


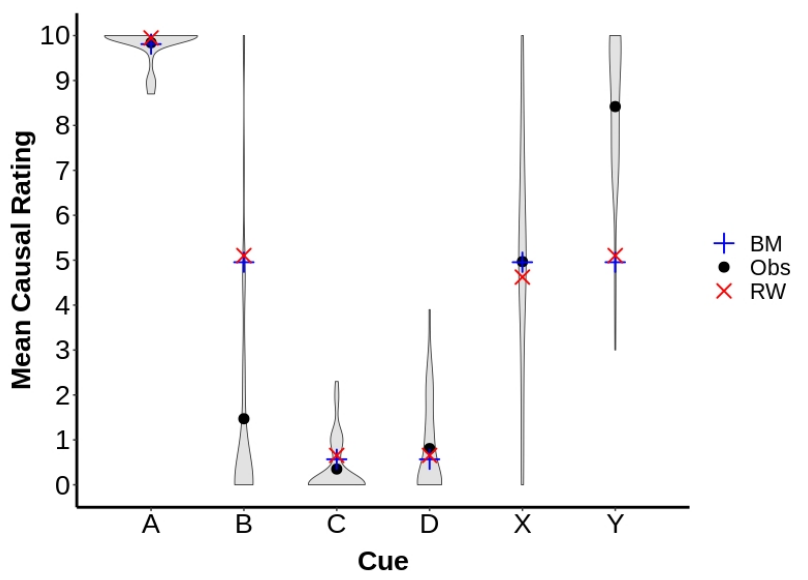

FIgure 1: Predicted versus observed test stage ratings for unmodified Rescorla \& Wagner (RW) model, and Bush \& Mosteller (BM) model, against observed data (Obs), following $A+/ A X+B-/ B Y+C-/ C D-$ training. The violin plot represents the distribution of the observed data using a method comparable to Hintze \& Nelson (1998). The distribution of the predicted data is not represented, as it was negligible.

\subsection{Modifying the Rescorla-Wagner model}

The Rescorla and Wagner (1972) model was originally developed as an account of non-human animal learning. In that context, it makes sense for the associative strength of cues to start at zero, because animals such as rats would not have learned a response to previously non-encountered cues. However, in our human blocking experiment, it seems unlikely that a novel cue would produce an associative strength of zero, since participants would lack sufficient information to determine whether or not it is a cause of stomach ache. An associative strength of zero should result in the production of low causal ratings, so a more intuitive response would be for participants to provide novel cues with an intermediate rating (for example 5 on a scale running from 0-10), reflecting their uncertainty about their causal status. This is supported by Spicer et al. (under review), in an experiment where a novel cue at test was assigned an intermediate rating of 4.85 on a $0-10$ likelihood scale. Negative associative strengths are also possible, meaning that a value between 0 and 1 is not strictly intermediate. However, the acquisition of negative associative strengths to cues (i.e. inhibition) is difficult to achieve in human predictive learning experiments using foods as cues (e.g. Zaksaite \& Jones, 2019). This is presumably because eating one food would not typically prevent an allergic reaction from being caused by another food. Therefore, an associative strength of 0.5 was regarded as an appropriate representation of participants' uncertainty.

We conducted our model fitting procedure on the blocking data a second time, using modified versions of both models, in which the initial associative strength of cues was an additional parameter for optimisation. The concept of non-zero starting associative strengths is not in itself novel (e.g. Gluck \& Bower, 1988); for example, non-zero initial strengths have been used to represent pre-training (Miller \& Shettleworth, 2007, Dupuis \& Dawson, 2013). However, using them as a representation of human uncertainty is novel. Moreover, the requirement of intermediate (non-zero) strengths in order for the Rescorla and Wagner (1972) model to explain a blocking experiment more effectively than the Bush and Mosteller (1951) model would be a novel finding. This concept has been discussed informally (e.g. Zaksaite, 2017) but not formally simulated. We chose to fully explore the parameter space, rather than setting this value at 0.5 , since if a substantially different value provided the best fit, it would indicate that our proposal was incorrect.

\subsection{Results from modified model implementa- tions}

If the Rescorla and Wagner (1972) model is modified so that the starting associative strength can be something other than zero, then it accounts for the results of our blocking experiment better than the equivalently modified Bush and Mosteller (1951) model. The modified Bush-Mosteller model produced a mean error of 0.04 and an $R^{2}$ of 0.93 . The modified Rescorla-Wagner model produced a mean error of 0.01 and an $R^{2}$ of 1.00 , therefore producing less error and a better adequacy of fit. Whilst the modified Bush-Mosteller model provided a better fit than the unmodified version (e.g. it predicted a difference between B and Y, because B declines in associative strength during the B- trials in Stage 1, while Y starts at an intermediate strength in Stage 2), the lack of a summed error term does not allow it to predict blocking. Figure 2 shows the predicted versus observed test cue ratings for both models.

As predicted, the best fitting initial associative strengths were at an intermediate value for both models ( 0.45 for BushMosteller and 0.43 for Rescorla-Wagner). This finding is consistent with the idea that participants assign intermediate ratings to cues that have an unknown causal status.

\section{Simulation set 2: Redundancy effect}

Next, we investigated whether our modified Rescorla and Wagner (1972) model could adequately capture a further psychological phenomenon that the unmodified model cannot; the redundancy effect (e.g. Jones \& Zaksaite, 2018; Jones et al., 2019; Uengoer et al., 2013; Uengoer et al., 2019). The training stage of a redundancy effect design incorporates blocking $(\mathrm{A}+\mathrm{AX}+)$ and a simple discrimination (BY+ CY-). Cue Y is referred to as an uncorrelated cue, because it appears in both a causal and a non-causal compound. 


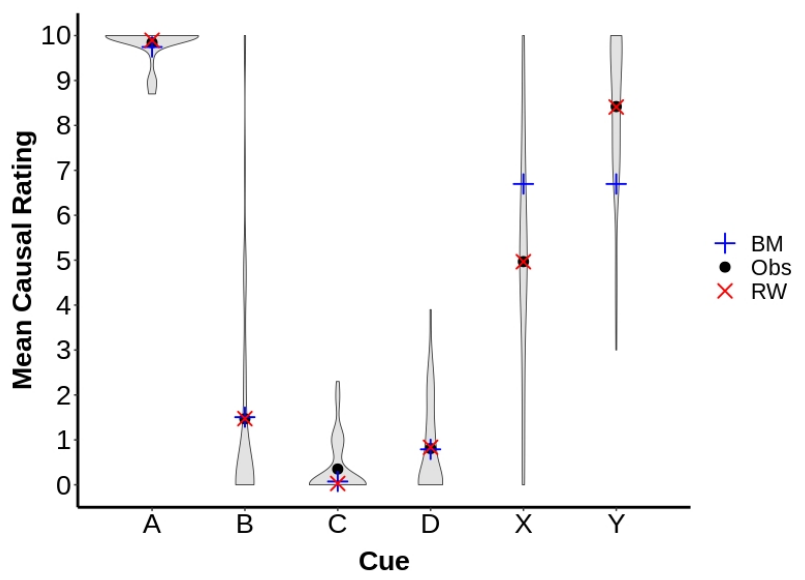

Figure 2: Predicted versus observed test stage ratings for modified Rescorla \& Wagner (RW) model, and Bush \& Mosteller (BM) model, against observed data (Obs), following $A+/ A X+B-/ B Y+C-/ C D$ - training. The violin plot represents the distribution of the observed data.

The redundancy effect is the observation of $\mathrm{X}$ being rated as a more likely cause of the outcome than Y. The blocked cue $(\mathrm{X})$ is typically given intermediate causal ratings at test, while the uncorrelated cue (Y) is typically given low causal ratings.

Rather than using a previously published data set, we collected a set of redundancy effect data (see Supplementary Materials). This was to provide a more diagnostic test stage than simply asking participants to provide likelihood ratings for the five single cues (A, B, C, X, Y). We also asked them to provide ratings for each of the ten possible compound cue pairs (that can be produced using these five individual cues). Importantly, the training participants received was equivalent to the training used in previous redundancy effect demonstrations $(\mathrm{A}+\mathrm{AX}+\mathrm{BY}+\mathrm{CY}-)$. However, having a wider set of cues in the test Stage meant that the two models could be 'stretched', by being required to fit a more complex set of test data.

\subsection{Results from modified model implementa- tions}

The modified Rescorla and Wagner (1972) model produced a mean error of 0.02 and an $R^{2}$ of 0.96 , indicating a good account of the dataset. This was better than the unmodified model, which produced a mean error of 0.03 and and $R^{2}$ of 0.83. The modified Bush and Mosteller (1951) model provided a poor account of the dataset $\left(R^{2}\right.$ of 0.63$)$, with no discernible improvement on the unmodified model $\left(R^{2}\right.$ of 0.62 ); this suggests that our modification is not simply leading to over-fitting from the inclusion of an additional parameter. Figure 3 shows the predicted versus observed
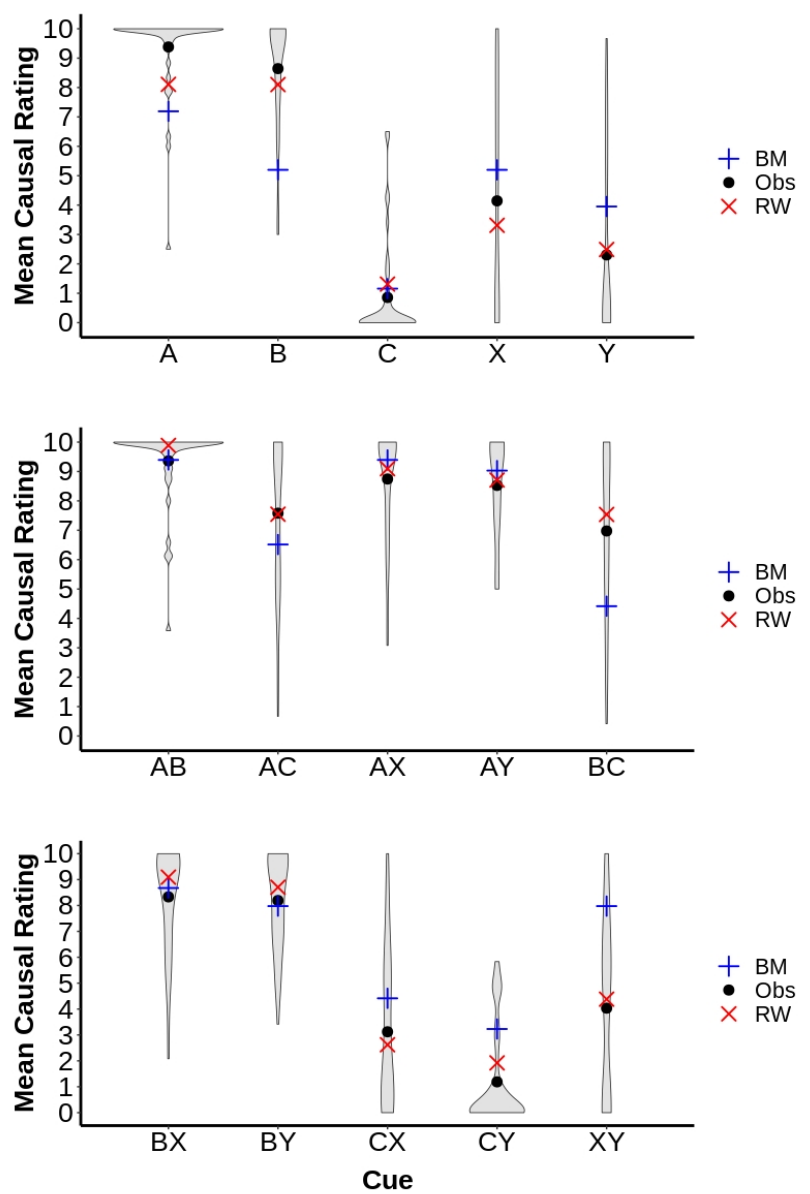

Figure 3: Predicted versus observed test stage ratings for modified Rescorla \& Wagner (RW) model, and Bush \& Mosteller (BM) model, against observed data (Obs), following $A+A X+B Y+C Y$ - training. The violin plot represents the distribution of the observed data.

test data for the modified models. The modified RescorlaWagner model captured the redundancy effect, although the size of the effect was somewhat underestimated. The BushMosteller model accounted for the redundancy effect itself, but not the full set of test data.

As with the blocking simulation, the best fitting initial associative strength was an intermediate value (0.62). It is notable that the value was slightly higher than for the blocking data. This could be because the outcome base rate during training (i.e. the proportion of trial types resulting in stomach ache versus no stomach ache) was higher. There is evidence from Jones et al. (2019) that participants' causal ratings of cues they are uncertain about are sensitive to the outcome base rate. If starting associative strength is an adequate representation of uncertainty about the status of novel cues, then the best-fitting starting associative strength should change in line with the outcome base rate. It is 
possible to test this idea by model fitting on a dataset in which the outcome base rate has been experimentally manipulated. This was the basis of our final model fitting procedure.

\section{Simulation set 3: Redundancy effect base rate manipulation}

A suitable dataset was already available for the final model fitting procedure. Jones et al. (2019) reported a redundancy effect experiment, in which the outcome base rate was varied between different groups of human participants. Full experimental details are available in their paper and a brief summary is included in our Supplementary Materials. The test-stage likelihood ratings assigned to blocked cues were shown to vary in line with the outcome base rate. In both groups, the redundancy effect was observed, because the blocked cue $(\mathrm{X})$ was assigned higher ratings than the uncorrelated cue (Y). However, the rating for $\mathrm{X}$ was higher in the high base rate group. The manipulation was achieved by adding additional cues, so that either $25 \%$ or $75 \%$ of training trials resulted in stomach ache. To test the prediction that starting associative strength is sensitive to experimental base rate, this parameter was allowed to vary by condition. None of the other parameters were allowed to vary by condition. If correct, the modified Rescorla and Wagner (1972) model should provide a good fit to all the test cues for both groups, with a higher best-fitting initial associative strength in the $75 \%$ group than in the $25 \%$ group.

\subsection{Results from modified model implementa- tion}

The modified Rescorla and Wagner (1972) model produced a mean error of 0.03 . The $R^{2}$ was 0.98 in both the $25 \%$ and $75 \%$ base rate groups, indicating a good fit. As predicted, the best fitting initial associative strength was higher in the high base rate group (0.51) than in the low base rate group (0.39). Our initial associative strength parameter appears to provide one reasonable way of representing participants' uncertainty about the causal status of novel cues. Of course, real participants, unlike our simulation, need to experience at least a few trials in order to become sensitive to base rate. Thus, using initial starting weights to model the effects of outcome base rate is necessarily a simplification of the mental operations involved. Figure 4 shows the predicted versus observed test stage ratings for the $25 \%$ and $75 \%$ groups. The modified Rescorla-Wagner model was able to capture the redundancy effect in both conditions. It also captured the labile nature of blocked cue $\mathrm{X}$, although the effect of the base rate on $\mathrm{X}$ is slightly underestimated.
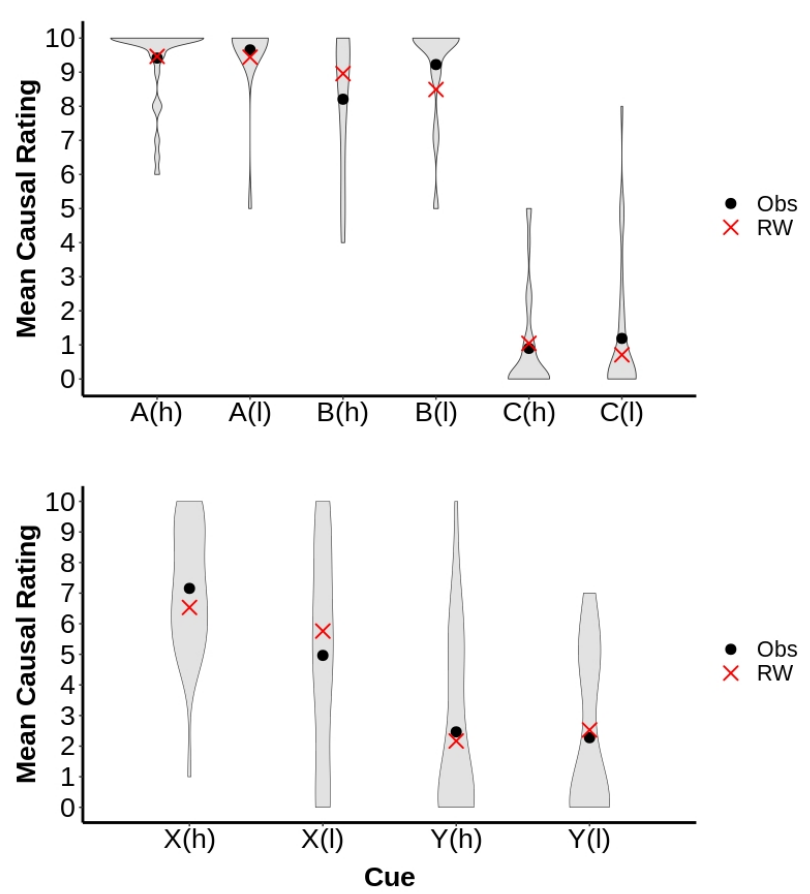

Figure 4: Predicted versus observed test stage ratings for modified Rescorla-Wagner (RW) model, against observed data (Obs), fitting to the high (h) and low (I) base rate groups, following $A+A X+B Y+C Y$ - training (intermixed with additional cues used to manipulate the base rate). The violin plot represents the distribution of the observed data.

\section{General Discussion}

\subsection{Summary of findings}

Contrary to intuition, the unmodified Rescorla and Wagner (1972) model provides no better an account of a standard forward cue-competition blocking experiment than the Bush and Mosteller (1951) model. However, if the former is modified, such that the initial associative strength of cues can be an intermediate value, then it does provide a better account than the equivalently modified Bush-Mosteller model. While the Rescorla-Wagner model can explain basic cue competition, it needs modification to be able fit the full experimental results; and can then do so almost perfectly. The modified Rescorla-Wagner model is also able to adequately account for the redundancy effect (e.g. Uengoer et al, 2013), which the unmodified model cannot. Additionally, the initial associative strength of the simulated cues was shown to vary with the outcome base rate, supporting the suggestion that intermediate initial associative strengths can be used to represent uncertainty about novel cues. This is consistent with the experimental findings of Jones et al. (2019), in that the likelihood ratings assigned to cues with an uncertain causal status are influenced by the outcome base rate. The best 
fitting initial associative strength may also be influenced by the range of plausible associative strengths within specific experimental scenarios. The experiments in this paper all used a food allergy scenario, in which inhibition was unlikely, bounding plausible associative strengths between 0 and 1. However, in a scenario where inhibition is possible, associative strengths could range from -1 to +1 , making 0 the intermediate value. This could be tested with a modified scenario (e.g. pharmaceutical drugs as cues), and a design with some cues explicitly trained as inhibitors.

\subsection{Fitting the full results}

We have taken the position in this article that it is important to fit the full results of an experiment, rather than a handselected subset of cues. One might argue that this approach could lead to trivial features of the experiment becoming crucial in model comparison. However, it seems to us that a cue cannot be both theoretically trivial and crucial in model comparison. For example, a cue might be theoretically trivial in the sense that all models inevitably predict responding to that cue correctly. If that is the case, then this cue cannot affect which of two competing models best fits the data inclusion of the trivial cue cannot change the winner of the contest. Conversely, a cue might affect the outcome of a model comparison to the extent that different models make different predictions about it. Such a cue is of theoretical importance, as it allows us to distinguish between different models. One might argue that experiments sometimes include cues that the models were not intended to explain, and thus including those cues in a model comparison is unfair. In such cases, it seems to us that the onus is on the proponents of the models to specify which sub-components of an experiment the model should not be expected to explain.

\subsection{Other accounts}

Vogel and Wagner (2016) suggested an alternative approach using the Rescorla and Wagner (1972) model that also accommodates the redundancy effect. In their approach, common elements are added to the stimulus representation. While this approach has been shown to accommodate the basic redundancy effect, there is evidence that it cannot adequately account for the effect of varying the outcome base rate on the redundancy effect (Jones et al., 2019). Nevertheless, further investigation of both approaches, across a range of phenomena, would be a fruitful direction for future research. The importance of making broad relative adequacy comparisons of models has been previously emphasised within the literature (Wills \& Pothos, 2012; Wills et al., 2017). Further research could also investigate a role for context in explaining the data (e.g. Bouton, 2010). Both minor experimental variations (e.g. different types of control used for blocking), and more substantially different designs, might produce different model comparison results. It would also be useful to know whether other associative models could account for these results (e.g. Gershman, 2015; Kokkola et al., 2019; McLaren \& Mackintosh, 2000, 2002; Schmajuk et al., 1996; Wagner, 1981); with or without modifications to the initial associative strengths.

\subsection{Extended test sets}

Instead of model fitting to a redundancy effect dataset only incorporating five single cues at test, we used an expanded set of test cues (Simulation Set 2). Given the high adequacy of fit ( $R^{2}$ value 0.96$)$ observed for the modified Rescorla and Wagner (1972) model, a further fitting procedure conducted on a dataset incorporating only five single test cues could not produce a fit any worse than this. Whilst there is some scope for the Bush and Mosteller (1951) model to produce a better fit with fewer test cues, the best this could result in is both models producing a comparably good fit as each other. In this scenario, the modified Rescorla-Wagner model would still provide the best account across the phenomena considered in this paper. We suggest that future predictive learning experiments should incorporate extended test sets, since this may provide a more diagnostic test of the relative adequacy of models. We welcome further debate and discussion of this suggestion.

\subsection{Open science for formal models}

Our simulations also demonstrate the value of thoroughly investigating the parameter space of formal models. Formal simulations are becoming more common in the study of human predictive learning, but one possible barrier is the apparent lack of a common open framework, in which models, phenomena and simulations can be easily assessed and compared. However, options are available, such as ALTSim (Thorwart et al. 2009). ALTSim does not allow for parameter space optimisation, but it does allow initial associative strengths to be set to values other than zero. The model implementations reported in the current paper used the free and open source package catlearn (Wills et al., 2020), which is available to download in the open-source $\mathrm{R}$ environment ( $\mathrm{R}$ Core Team, 2021). catlearn includes a number of model implementations, including Rescorla and Wagner (1972), Bush and Mosteller (1951), EXIT (Kruschke, 2001), and COVIS (Ashby et al., 1998). It is an extensible framework to which more models can be added.

\subsection{Conclusion}

Our simulations show that intermediate starting associative strengths are needed for the Rescorla and Wagner (1972) model to fit the results of a simple forward cue competition experiment better than the Bush and Mosteller (1951) model. 
Contrary to intuition, both models perform equally poorly in the absence of this change. Furthermore, this simple change allows the Rescorla-Wagner model to account for both the redundancy effect, and the effect of base rate on the redundancy effect.

\section{Author contributions}

SGS (lead author): Co-contributor to the rationale, design of the experiments, and theoretical basis of the simulations. Programmed the simulations and experiments. Co-programmed the model implementations. Collected and analysed the data. Wrote up the simulations and experiments. AJW: Co-contributor to the rationale, design of the experiments, and theoretical basis of the simulations. Consulted on analysis and write up of simulations and experiments. PMJ: Contributed to rationale, interpretation and write up. CJM: Contributed to interpretation and write up. LD: Coprogrammed the model implementations.

\section{References}

Ashby, F. G., Alfonso-Reese, L. A., \& Waldron, E. M. (1998). A neuropsychological theory of multiple systems in category learning. Psychological Review, 105(3), 442-481. https://doi.org/10.1037/0033-295x.105.3.442

Bouton, M. E. (2010). The multiple forms of "context" in associative learning theory. In B. Mesquita, L. F. Barrett, \& E. R. Smith (Eds.), The mind in context (p. 233-258). Guilford Press. http://doi.org/10.1101/1m.493707

Bush, R. R., \& Mosteller, F. (1951). A mathematical model for simple learning. Psychological Review, 58, 313-323. https://doi.org/10.1007/978-0-387-44956-2_12

Dickinson, A., Shanks, D., \& Evenden, J. (1984). Judgement of act-outcome contingency: The role of selective attribution. The Quarterly Journal of Experimental Psychology, 36(1), 29-50. https://doi.org/10.1080/ 14640748408401502

Dupuis, B., \& Dawson, M. R. (2013). Differentiating models of associative learning: Reorientation, superconditioning, and the role of inhibition. Journal of Experimental Psychology: Animal Behavior Processes, 39(3), 273. https://doi.org/10.1037/a0032174

Gershman, S. J. (2015). A unifying probabilistic view of associative learning. PLoS Computational Biology, 11(11). https://doi.org/10.1371/journal.pcbi.1004567

Gluck, M. A., \& Bower, G. H. (1988). From conditioning to category learning: an adaptive network model. Journal of Experimental Psychology: General, 117(3), 227-247. https://doi.org/10.1037/0096-3445.117.3.227

Jones, P. M., \& Zaksaite, T. (2018). The redundancy effect in human causal learning: no evidence for changes in selective attention. Quarterly Journal of Experimental
Psychology, 71(8), 1748-1760. https://doi.org/10.1080/ 17470218.2017.1350868

Jones, P. M., Zaksaite, T., \& Mitchell, C. J. (2019). Uncertainty and blocking in human causal learning. Journal of Experimental Psychology: Animal Learning and Cognition, 45(1), 111-124. https://doi.org/10.1037/ xan0000185

Kamin, L. J. (1969). Selective association and conditioning. In N. J. Mackintosh \& W. K. Honig (Eds.), Fundamental Issues in Associative Learning (pp. 42-64). Halifax, Canada: Dalhousie University Press.

Kokkola, N. H., Mondragón, E., \& Alonso, E. (2019). A double error dynamic asymptote model of associative learning. Psychological Review, 126(4), 506. https: //doi.org/10.1101/210674d

Kruschke, J. K. (2001). Toward a unified model of attention in associative learning. Journal of Mathematical Psychology, 45(6), 812-863. https://doi.org/10.1006/jmps.2000. 1354

McLaren, I. P. L., \& Mackintosh, N. J. (2000). An elemental model of associative learning: I. Latent inhibition and perceptual learning. Animal Learning \& Behavior, 28(3), 211-246. https://doi.org/10.3758/BF03200258

McLaren, I. P. L., \& Mackintosh, N. J. (2002). Associative learning and elemental representation: II. Generalization and discrimination. Animal Learning \& Behavior, 30(3), 177-200. https://doi.org/10.3758/BF03192828

Miller, R. R., \& Matute, H. (1996). Biological significance in forward and backward blocking: Resolution of a discrepancy between animal conditioning and human causal judgment. Journal of Experimental Psychology: General, 125(4), 370-386. https://doi.org/10.1037/0096-3445.125. 4.370

Miller, N. Y., \& Shettleworth, S. J. (2007). Learning about environmental geometry: An associative model. Journal of Experimental Psychology: Animal Behavior Processes, 33(3), 191-212. https://doi.org/10.1037/00977403.33.3.191

Mitchell, C. J., \& Lovibond, P. F. (2002). Backward and forward blocking in human electrodermal conditioning: Blocking requires an assumption of outcome additivity. Quarterly Journal of Experimental Psychology, 55B(4), 311-329. https://doi.org/10.1080/02724990244000025

Nosofsky, R. M., Gluck, M. A., Palmeri, T. J., McKinley, S. C., \& Glauthier, P. (1994). Comparing modes of rulebased classification learning: A replication and extension of Shepard, Hovland, and Jenkins (1961). Memory \& Cognition, 22, 352-369.

R Core Team. (2021). R: A language and environment for statistical computing. www.r-project.org. (Version 4.0.4)

Rescorla, R. A., and Wagner, A. R. (1972). A theory of Pavlovian conditioning: Variations in the effectiveness of reinforcement and nonreinforcement. In A. H. Black \& W. F. Prokasy (Eds.), Classical conditioning II: Cur- 
rent theory and research (pp. 64-99). New York, NY: Appleton-Century-Crofts. ISBN: 0390718017.

Schmajuk, N. A., Lam, Y.-W., \& Gray, J. A. (1996). Latent inhibition: A neural network approach. Journal of Experimental Psychology: Animal Behavior Processes, 22(3), 321-349. https://doi.org/10.1037/0097-7403.22.3.321

Shanks, D. R. (1985). Forward and backward blocking in human contingency judgement. Quarterly Journal of Experimental Psychology, 37B(1), 1-21. https: //doi.org/10.1080/14640748508402082

Spicer, S. G., Mitchell, C. J., Wills, A. J., Blake. K. L., and Jones, P. M. (under review). Theory protection: do humans protect existing associative links? Journal of Experimental Psychology: Animal Learning and Cognition.

Thorwart, A., Schultheis, H., König, S. \& Lachnit, H. (2009). ALTSim: A MATLAB simulator for current associative learning theories. Behavior Research Methods, 41, 29-34. https://doi.org/10.3758/brm.41.1.29

Uengoer, M., Lotz, A., Pearce, J. M., (2013). The fate of redundant cues in human predictive learning. Journal of Experimental Psychology: Animal Behaviour Processes, 39(4), 323-333. https://doi.org/10.1037/a0034073

Uengoer, M., Dwyer, D. M., Koenig, S., \& Pearce, J. M. (2019). A test for a difference in the associability of blocked and uninformative cues in human predictive learning. Quarterly Journal of Experimental Psychology, 72(2), 222-237.

Wagner, A. R. (1981). SOP: A model of automatic memory processing in animal behavior. In N. E. Spear \& R.R. Miller. Information Processing in Animals: Memory Mechanisms (pp. 5-47). ISBN: 9780898591576.

Wills, A. J., \& Pothos, E. M. (2012). On the adequacy of current empirical evaluations of formal models of categorization. Psychological Bulletin, 138(1), 102-125. https://doi.org/10.1037/a0025715

Wills, A. J., O'Connell, G., Edmunds, C. E., \& Inkster, A. B. (2017). Progress in modeling through distributed collaboration: Concepts, tools and category-learning examples. Psychology of Learning and Motivation, 66, 79-115. https://doi.org/10.1016/bs.plm.2016.11.007

Wills, A. J., Dome, L., Edmunds C. E., Honke, G., Inkster, A. B., Schlegelmilch, R., \& Spicer, S. G. (2020). catlearn: Formal Psychological Models of Categorization and Learning. https://CRAN.R-project.org/package= catlearn. $R$ package version 0.8 .

Zaksaite, G. (2017). The Redundancy Effect in Human Causal Learning: Attention, Uncertainty, And Inhibition. Doctoral dissertation, University of Plymouth.

Zaksaite, T., \& Jones, P. M. (2019). The redundancy effect is related to a lack of conditioned inhibition: Evidence from a task in which excitation and inhibition are symmetrical. Quarterly Journal of Experimental Psychology, 73(2), 260-278. https://doi.org/10.1177/1747021819878430 